\title{
Parabolic Triangles, Poles and Centroid Relations
}

\section{Parabolic Triangles, Poles and Centroid Relations ABSTRACT}

We investigate affine properties of centroids formed by three points on a parabola together with the polar triangle formed from the tangents. And we make a wide ranging conjecture about the extension of these results to general conics.

Key words: affine geometry, parabola, centroids, conics MSC2010: 51N20, 14H50, 14Rxx

\section{Introduction}

The parabola has been intensively studied since antiquity, see for example [2], [4], [5], [6], [7] and [8]. In this paper we explore purely affine properties of a parabola, and in particular the configuration formed by three points $A_{1}, A_{2}$ and $A_{3}$ on the parabola, and the corresponding duals, or poles $X_{1}, X_{2}$ and $X_{3}$ of the three lines formed by those points. We are particularly interested in the relations between centroids of various triangles formed by the six points $A_{1}, A_{2}, A_{3}, X_{1}, X_{2}$ and $X_{3}$. An example of such a result is the following, which was discussed by D. Liu [3].

Theorem: If $G_{123}$ is the centroid of the triangle $\overline{A_{1} A_{2} A_{3}}$ and $G^{123}$ is the centroid of the triangle $\overline{X_{1} X_{2} X_{3}}$, then the line $G_{123} G^{123}$ is parallel to the axis direction of the parabola.

We note that while a distinguished axis of a parabola is a metrical object, the axis direction itself is not, as it is simply the direction corresponding to the unique point at infinity on the parabola, so this is indeed a result purely of affine geometry-independent of any metrical, such as Euclidean, structure.

\section{Trokuti upisani paraboli, polovi i težišta}

\section{SAŽETAK}

Istražujemo afina svojstva težišta koja određuju tri točke na paraboli i vrhovi njihovog polarnog trokuta. Donosimo pretpostavku o proširenju dobivenih rezultata na sve konike.

Ključne riječi: afina geometrija, parabola, težišta, konike

We will show that in fact these six points support a rich fabric of results. In the final paragraph of the paper, we will propose a large-scale generalization of this phenomenon: in fact we conjecture that all the theorems in fact hold for a generic conic in the plane. However our proofs depend heavily on explicit computation, following the framework of [1]. We consider the tabulation of various points and lines a useful tool for further additional research in this direction.

\section{Affine geometry and the standard para- bola}

We will find it convenient to use projective coordinates for points and lines, see for example [2] and [7], even in the affine situation. The underlying number system is a general field, and our arguments will try to avoid any particular assumptions about this field.

An affine point is an expression of the form $[x, y]$, and in projective coordinates it will here be written $A=[1: x: y]$. A general projective point is then $[z: x: y]$ where by agree- 
ment we can re-scale by any non-zero number, so that

$[z: x: y]=[r z: r x: r y]$

for any $r \neq 0$.

An affine line is a proportion $(c: a: b)$ of numbers, with at least one of $a$ and $b$ non-zero. The point $A \equiv[x, y]$ lies on the line $l \equiv(c: a: b)$ precisely when

$c+a x+b y=0$

which we also call the equation of the line. Equivalently we say $l$ passes through $A$, or that $l$ and $A$ are incident. In projective coordinates, the point $[z: x: y]$ is incident with the line $(c: a: b)$ precisely when

$c z+a x+b y=0$

If $A_{1}=\left[z_{1}: x_{1}: y_{1}\right]$ and $A_{2}=\left[z_{2}: x_{2}: y_{2}\right]$ are two distinct points in projective coordinates then their join is the unique line $A_{1} A_{2}$ passing through them both, and in projective coordinates is formed from a cross product:

$A_{1} A_{2}=\left(x_{1} y_{2}-x_{2} y_{1}: y_{1} z_{2}-y_{2} z_{1}: x_{2} z_{1}-x_{1} z_{2}\right)$.

Similarly if $l_{1}=\left(k_{1}: l_{1}: m_{1}\right)$ and $l_{2}=\left(k_{2}: l_{2}: m_{2}\right)$ are two distinct lines in projective coordinates then their meet is the unique point $l_{1} l_{2}$ lying on them both, which is formed from the same cross product, and is

$l_{1} l_{2}=\left[l_{1} m_{2}-l_{2} m_{1}: k_{2} m_{1}-k_{1} m_{2}: k_{1} l_{2}-k_{2} l_{1}\right]$.

This approach treats points and lines symmetrically, and provides good motivation for us to use projective coordinates even in affine geometry. It also brings the crucial computations of joins and meets together. We will move freely from affine to projective coordinates as needed in this paper.

We recall various standard results from [1], reformulated in this projective framework, and simplified somewhat. The general parabola with equation

$y^{2}=4 \lambda x$

may be re-scaled by sending $[x, y]$ to $[\lambda x, \lambda y]$. in which case it becomes the simpler equation

$y^{2}=4 x$

or in projective coordinates

$y^{2}=4 x z$.

We will call this now the standard parabola, recognizing that the formulas of may be transformed to this simpler setting just by setting $\lambda=1$ throughout. Using projective coordinates, let $\left[1: t^{2}: 2 t\right]$ denote a general point on this parabola, and suppose that

$A \equiv\left[1: a^{2}: 2 a\right] \quad$ and $\quad B \equiv\left[1: b^{2}: 2 b\right]$

are two specific points on the parabola.

The chord $A B$ in Cartesian form is given in projective coordinates by a cross product, which after re-scaling is

$$
\begin{aligned}
& {\left[1, a^{2}, 2 a\right] \times\left[1, b^{2}, 2 b\right]} \\
& =(2 a b(a-b): 2(a-b):-(a-b)(a+b)) \\
& =(2 a b: 2:-a-b) .
\end{aligned}
$$

The tangent to the parabola at $A$ may be simply obtained by setting $a=b$ in the equation of the above chord, giving

$\left(2 a^{2}: 2:-2 a\right)=\left(a^{2}: 1:-a\right)$.

The external point of the chord $A B$ is the meet of the tangents at $A$ and $B$, which after a cross product and re-scaling is

$$
\begin{aligned}
& \left(a^{2}, 1,-a\right) \times\left(b^{2}, 1,-b\right) \\
& =\left[a-b: a^{2} b-a b^{2}: a^{2}-b^{2}\right] \\
& =[1: a b: a+b] .
\end{aligned}
$$

\section{Parabolic triangles and Liu's result}

Let $A_{1}, A_{2}$ and $A_{3}$ be three distinct points on the standard parabola, forming what we call a parabolic triangle $\overline{A_{1} A_{2} A_{3}}$ and which we denote by $\Delta_{123}$. Without loss of generality we assume that

$A_{1} \equiv\left[1: a_{1}^{2}: 2 a_{1}\right], A_{2} \equiv\left[1: a_{2}^{2}: 2 a_{2}\right], A_{3} \equiv\left[1: a_{3}^{2}: 2 a_{3}\right]$ with the numbers $a_{1}, a_{2}$ and $a_{3}$ distinct. The lines of $\Delta_{123}$ are then

$$
\begin{aligned}
& A_{1} A_{2}=\left(2 a_{1} a_{2}: 2:-a_{1}-a_{2}\right) \\
& A_{2} A_{3}=\left(2 a_{2} a_{3}: 2:-a_{2}-a_{3}\right) \\
& A_{1} A_{3}=\left(2 a_{3} a_{1}: 2:-a_{3}-a_{1}\right) .
\end{aligned}
$$

The centroid of $\overline{A_{1} A_{2} A_{3}}$ is, switching to affine coordinates and averaging,

$$
\begin{aligned}
G_{123} & =\frac{1}{3}\left[a_{1}^{2}, 2 a_{1}\right]+\frac{1}{3}\left[a_{2}^{2}, 2 a_{2}\right]+\frac{1}{3}\left[a_{3}^{2}, 2 a_{3}\right] \\
& =\left[\frac{1}{3} a_{1}^{2}+\frac{1}{3} a_{2}^{2}+\frac{1}{3} a_{3}^{2}, \frac{2}{3} a_{1}+\frac{2}{3} a_{2}+\frac{2}{3} a_{3}\right] \\
& =\left[3: a_{1}^{2}+a_{2}^{2}+a_{3}^{2}: 2\left(a_{1}+a_{2}+a_{3}\right)\right] .
\end{aligned}
$$

The external points, or poles, of the sides $\overline{A_{2} A_{3}}, \overline{A_{1} A_{3}}$ and $\overline{A_{1} A_{2}}$ are respectively

$X_{1} \equiv\left[1: a_{2} a_{3}: a_{2}+a_{3}\right], X_{2} \equiv\left[1: a_{1} a_{3}: a_{1}+a_{3}\right]$,

$X_{3} \equiv\left[1: a_{1} a_{2}: a_{1}+a_{2}\right]$. 
We denote the external triangle $\overline{X_{1} X_{2} X_{3}}$ by $\Delta^{123}$. Its centroid is

$$
\begin{aligned}
& G^{123} \\
& =\frac{1}{3}\left[a_{2} a_{3}, a_{2}+a_{3}\right]+\frac{1}{3}\left[a_{1} a_{3}, a_{1}+a_{3}\right]+\frac{1}{3}\left[a_{1} a_{2}, a_{1}+a_{2}\right] \\
& =\left[\frac{1}{3} a_{1} a_{2}+\frac{1}{3} a_{2} a_{3}+\frac{1}{3} a_{1} a_{3}, \frac{2}{3} a_{1}+\frac{2}{3} a_{2}+\frac{2}{3} a_{3}\right] \\
& =\left[3: a_{1} a_{2}+a_{2} a_{3}+a_{1} a_{3}: 2\left(a_{1}+a_{2}+a_{3}\right)\right] .
\end{aligned}
$$

We now give a proof of the observation of $\mathrm{D}$. Liu.

Theorem 1 The line $G_{123} G^{123}$ is always parallel to the axis direction of the parabola, and in this case has equation

$y=\frac{2}{3}\left(a_{1}+a_{2}+a_{3}\right)$.

Proof. The axis direction for the standard parabola is just the $x$-axis, so this is the statement that $G_{123} G^{123}$ is horizontal. We compute that

$$
\begin{aligned}
& G_{123} G^{123} \\
& =\left[3, a_{1}^{2}+a_{2}^{2}+a_{3}^{2}, 2\left(a_{1}+a_{2}+a_{3}\right)\right] \\
& \quad \quad \times\left[3, a_{1} a_{2}+a_{2} a_{3}+a_{1} a_{3}, 2\left(a_{1}+a_{2}+a_{3}\right)\right] \\
& =\left(2\left(a_{1}+a_{2}+a_{3}\right): 0:-3\right)
\end{aligned}
$$

with equation

$y=\frac{2}{3}\left(a_{1}+a_{2}+a_{3}\right)$.

Another approach would be to compute the vector

$$
\begin{aligned}
& \overrightarrow{G_{123} G^{123}} \\
& =\frac{1}{3}\left[a_{1} a_{2}+a_{2} a_{3}+a_{1} a_{3}, 2\left(a_{1}+a_{2}+a_{3}\right)\right] \\
& \quad \quad-\frac{1}{3}\left[a_{1}^{2}+a_{2}^{2}+a_{3}^{2}, 2\left(a_{1}+a_{2}+a_{3}\right)\right] \\
& =-\frac{1}{3}\left(a_{1}^{2}+a_{2}^{2}+a_{3}^{2}-a_{1} a_{2}-a_{2} a_{3}-a_{1} a_{3}\right)(1,0) .
\end{aligned}
$$

\section{More general centroids}

The six points $A_{1}, A_{2}, A_{3}, X_{1}, X_{2}$ and $X_{3}$ determine also mixed triangles, such as for example

$$
\overline{A_{1} A_{2} X_{3}}=\Delta_{12}^{3} \quad \text { or } \quad \overline{A_{1} X_{2} X_{3}}=\Delta_{1}^{23}
$$

where the lower indices record $A_{i}$ vertices, and the raised indices record $X_{j}$ vertices, and where we agree to arrange indices always in ascending order.
Define $G_{i j}^{k}$ to be the centroid of $\Delta_{i j}^{k}$, for all allowed index combinations $i, j$ and $k$. Then we may compute, using projective coordinates, that

$$
\begin{aligned}
& G_{12}^{1}=\left[3: a_{1}^{2}+a_{2}^{2}+a_{2} a_{3}: 3 a_{2}+2 a_{1}+a_{3}\right] \\
& G_{12}^{2}=\left[3: a_{1}^{2}+a_{2}^{2}+a_{1} a_{3}: 3 a_{1}+2 a_{2}+a_{3}\right] \\
& G_{23}^{2}=\left[3: a_{2}^{2}+a_{3}^{2}+a_{1} a_{3}: 3 a_{3}+2 a_{2}+a_{1}\right] \\
& G_{23}^{3}=\left[3: a_{2}^{2}+a_{3}^{2}+a_{1} a_{2}: 3 a_{2}+2 a_{3}+a_{1}\right] \\
& G_{13}^{1}=\left[3: a_{1}^{2}+a_{3}^{2}+a_{2} a_{3}: 3 a_{3}+2 a_{1}+a_{2}\right] \\
& G_{13}^{3}=\left[3: a_{1}^{2}+a_{3}^{2}+a_{1} a_{2}: 3 a_{1}+2 a_{3}+a_{2}\right]
\end{aligned}
$$

while

$$
\begin{aligned}
& G_{1}^{12}=\left[3: a_{1} a_{3}+a_{2} a_{3}+a_{1}^{2}: 3 a_{1}+2 a_{3}+a_{2}\right] \\
& G_{2}^{12}=\left[3: a_{1} a_{3}+a_{2} a_{3}+a_{2}^{2}: 3 a_{2}+2 a_{3}+a_{1}\right] \\
& G_{1}^{13}=\left[3: a_{1} a_{2}+a_{2} a_{3}+a_{1}^{2}: 3 a_{1}+2 a_{2}+a_{3}\right] \\
& G_{3}^{13}=\left[3: a_{1} a_{2}+a_{2} a_{3}+a_{3}^{2}: 3 a_{3}+2 a_{2}+a_{1}\right] \\
& G_{2}^{23}=\left[3: a_{1} a_{3}+a_{1} a_{2}+a_{2}^{2}: 3 a_{2}+2 a_{1}+a_{3}\right] \\
& G_{3}^{23}=\left[3: a_{1} a_{2}+a_{1} a_{3}+a_{3}^{2}: 3 a_{3}+2 a_{1}+a_{2}\right] .
\end{aligned}
$$

In fact the definitions also make sense for a degenerate triangle such as $\overline{A_{1} X_{2} X_{3}}$ : even though these points are collinear, their centroid $G_{1}^{23}$ is well-defined and altogether we have six such points, namely

$$
\begin{aligned}
G_{23}^{1} & =\left[3: a_{2}^{2}+a_{3}^{2}+a_{2} a_{3}: 3\left(a_{2}+a_{3}\right)\right] \\
G_{1}^{23} & =\left[3: a_{1}\left(a_{1}+a_{2}+a_{3}\right): 4 a_{1}+a_{2}+a_{3}\right] \\
G_{13}^{2} & =\left[3: a_{1}^{2}+a_{3}^{2}+a_{1} a_{3}: 3\left(a_{1}+a_{3}\right)\right] \\
G_{2}^{13} & =\left[3: a_{2}\left(a_{1}+a_{2}+a_{3}\right): a_{1}+4 a_{2}+a_{3}\right] \\
G_{12}^{3} & =\left[3: a_{1}^{2}+a_{2}^{2}+a_{1} a_{2}: 3\left(a_{1}+a_{2}\right)\right] \\
G_{3}^{12} & =\left[3: a_{3}\left(a_{1}+a_{2}+a_{3}\right): a_{1}+a_{2}+4 a_{3}\right] .
\end{aligned}
$$

Theorem 2 For any distinct indices $1 \leq i<j \leq 3$

$$
\overrightarrow{G_{i}^{i j} G_{j}^{i j}}=\frac{1}{3} \overrightarrow{A_{i} A_{j}} .
$$

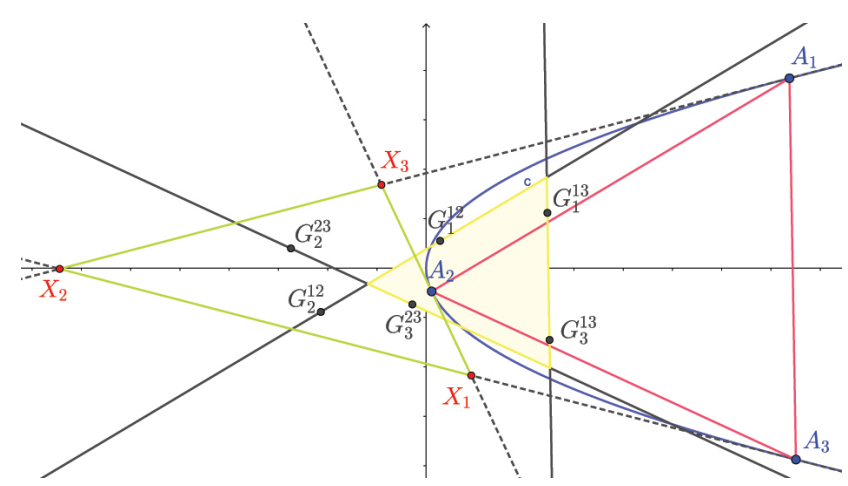

Figure 1: Mixed centroids of a parabolic triangle 
Proof. We consider the case $i=1$ and $j=2$, the other cases are similar. Using affine coordinates, we have that

$$
\begin{aligned}
\overrightarrow{A_{1} A_{2}} & =A_{2}-A_{1}=\left[a_{2}^{2}, 2 a_{2}\right]-\left[a_{1}^{2}, 2 a_{1}\right] \\
& =\left(a_{2}^{2}-a_{1}^{2}, 2 a_{2}-2 a_{1}\right) \\
& =\left(a_{2}-a_{1}\right)\left(a_{1}+a_{2}, 2\right) .
\end{aligned}
$$

But also

$$
\begin{aligned}
\overrightarrow{G_{1}^{12} G_{2}^{12}}= & \frac{1}{3}\left[a_{1} a_{3}+a_{2} a_{3}+a_{2}^{2}, 3 a_{2}+2 a_{3}+a_{1}\right] \\
& -\frac{1}{3}\left[a_{1} a_{3}+a_{2} a_{3}+a_{1}^{2}, 3 a_{1}+2 a_{3}+a_{2}\right] \\
= & \frac{1}{3}\left(a_{2}^{2}-a_{1}^{2}, 2 a_{2}-2 a_{1}\right) \\
= & \frac{1}{3}\left(a_{2}-a_{1}\right)\left(a_{1}+a_{2}, 2\right) .
\end{aligned}
$$

The result follows.

Theorem 3 Let $I_{1}$ be the meet of the lines $G_{1}^{12} G_{2}^{12}$ and $G_{1}^{13} G_{3}^{13}, I_{2}$ be the meet of the lines $G_{1}^{12} G_{2}^{12}$ and $G_{2}^{23} G_{3}^{23}$, and $I_{3}$ be the meet of the lines $G_{1}^{13} G_{3}^{13}$ and $G_{2}^{23} G_{3}^{23}$. Then for $1 \leq i<j \leq 3$

$\vec{I}_{i} I_{j}=\frac{1}{2} \overrightarrow{A_{i} A_{j}}$.

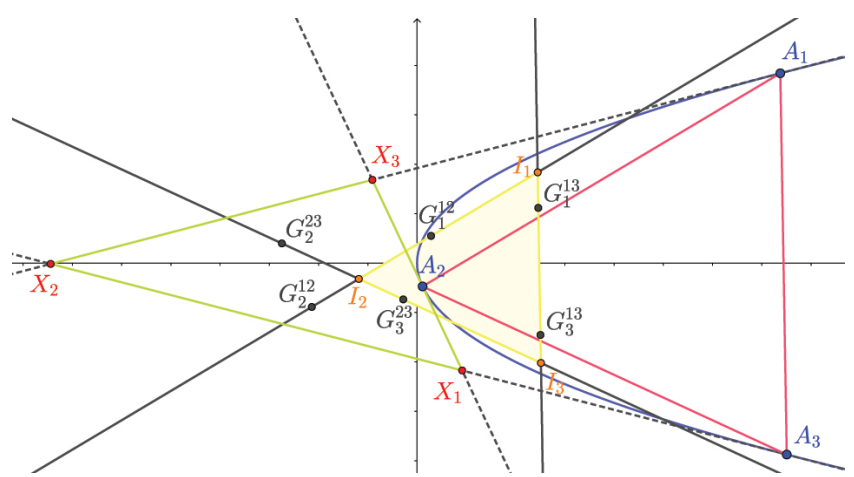

Figure 2: An affinely similar triangle $\overline{I_{1} I_{2} I_{3}}$ to $\overline{A_{1} A_{2} A_{3}}$

Proof. The equations of the relevant lines through the centroids are

$$
\begin{aligned}
& G_{1}^{12} G_{2}^{12}=\left(a_{1}^{2}+a_{2}^{2}+4 a_{1} a_{2}: 6:-3\left(a_{1}+a_{2}\right)\right) \\
& G_{2}^{23} G_{3}^{23}=\left(a_{2}^{2}+a_{3}^{2}+4 a_{2} a_{3}: 6:-3\left(a_{2}+a_{3}\right)\right) \\
& G_{3}^{13} G_{1}^{13}=\left(a_{3}^{2}+a_{1}^{2}+4 a_{3} a_{1}: 6:-3\left(a_{3}+a_{1}\right)\right) .
\end{aligned}
$$

Then the meets of these lines can calculated as follows

$$
\begin{aligned}
I_{1} & =\left(G_{1}^{12} G_{2}^{12}\right)\left(G_{1}^{13} G_{3}^{13}\right) \\
& =\left[6: a_{1} a_{2}+a_{2} a_{3}+a_{3} a_{1}+3 a_{1}^{2}: 2\left(a_{2}+a_{3}+4 a_{1}\right)\right] \\
I_{2} & =\left(G_{1}^{12} G_{2}^{12}\right)\left(G_{2}^{23} G_{3}^{23}\right) \\
& =\left[6: a_{1} a_{2}+a_{2} a_{3}+a_{3} a_{1}+3 a_{2}^{2}: 2\left(a_{3}+a_{1}+4 a_{2}\right)\right] \\
I_{3} & =\left(G_{1}^{13} G_{3}^{13}\right)\left(G_{2}^{23} G_{3}^{23}\right) \\
& =\left[6: a_{1} a_{2}+a_{2} a_{3}+a_{3} a_{1}+3 a_{3}^{2}: 2\left(a_{1}+a_{2}+4 a_{3}\right)\right] .
\end{aligned}
$$

Then for the case $i=1$ and $j=2, \overline{I_{1} I_{2}}$ can be calculated using affine coordinates as

$$
\begin{aligned}
\overrightarrow{I_{1} I_{2}}= & I_{2}-I_{1} \\
= & \frac{1}{6}\left[a_{1} a_{2}+a_{2} a_{3}+a_{3} a_{1}+3 a_{2}^{2}, 2\left(a_{3}+a_{1}+4 a_{2}\right)\right] \\
& -\frac{1}{6}\left[a_{1} a_{2}+a_{2} a_{3}+a_{3} a_{1}+3 a_{1}^{2}, 2\left(a_{2}+a_{3}+4 a_{1}\right)\right] \\
= & \left(\frac{1}{2}\left(a_{2}^{2}-a_{1}^{2}\right),\left(a_{2}-a_{1}\right)\right) \\
= & \frac{1}{2}\left(a_{2}-a_{1}\right)\left(a_{1}+a_{2}, 2\right) .
\end{aligned}
$$

The result for $\overrightarrow{I_{1} I_{2}}$ follows, and the other cases are symmetrically similar.

Recall that in affine geometry the signed area of a triangle $\overrightarrow{A_{1} A_{2} A_{3}}$ with oriented sides $\overrightarrow{A_{1} A_{2}}=(a, b)$ and $\overrightarrow{A_{1} A_{3}}=$ $(c, d)$ is

$s\left(\overrightarrow{A_{1} A_{2} A_{3}}\right) \equiv \frac{1}{2}(a d-b c)$

again independent of any metrical structure.

Corollary 1 The triangles $\Delta_{123}$ and $\overline{I_{1} I_{2} I_{3}}$ have parallel sides and so are affinely similar, and so their signed areas satisfy

$s\left(\overrightarrow{A_{1} A_{2} A_{3}}\right): s\left(\overrightarrow{I_{1} I_{2} I_{3}}\right)=4: 1$.

Proof. This follows from the relation between the vectors making the sides of the triangles.

Theorem 4 The triangles $\overline{A_{1} A_{2} A_{3}}$ and $\overline{I_{1} I_{2} I_{3}}$ are perspective from the centroid $G^{123}$ of $\Delta^{123}$.

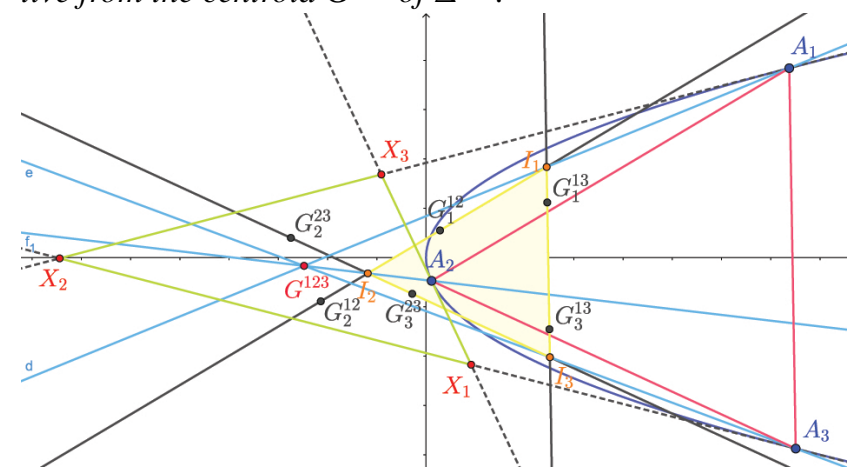

Figure 3: A centre of perspectivity $G^{123}$

Proof. Using our formula for joins we compute that

$$
\begin{aligned}
A_{1} I_{1}=\left(2\left(a_{1} a_{2} a_{3}-a_{1}^{3}\right): 2\right. & \left(a_{2}+a_{3}-2 a_{1}\right) \\
: & \left.3 a_{1}^{2}-a_{1} a_{2}-a_{2} a_{3}-a_{3} a_{1}\right) .
\end{aligned}
$$


To check that the centroid

$G^{123}=\left[3: a_{1} a_{2}+a_{2} a_{3}+a_{1} a_{3}: 2\left(a_{1}+a_{2}+a_{3}\right)\right)$

is incident with this line, verify that

$$
\begin{array}{r}
2\left(a_{1} a_{2} a_{3}-a_{1}^{3}\right) 3+2\left(a_{2}+a_{3}-2 a_{1}\right)\left(a_{1} a_{2}+a_{2} a_{3}+a_{1} a_{3}\right) \\
+\left(3 a_{1}^{2}-a_{1} a_{2}-a_{2} a_{3}-a_{3} a_{1}\right) 2\left(a_{1}+a_{2}+a_{3}\right)=0 .
\end{array}
$$

Since this argument also holds for $A_{2} I_{2}$ and $A_{3} I_{3}$, all three lines are concurrent at the centroid $G^{123}$.

In particular it follows that the triangles $\overline{I_{1} I_{2} I_{3}}$ and $\Delta_{123}$ are in perspective via the centroid $G^{123}$ of triangle $\Delta^{123}$.

Corollary 2 The points $I_{1}, I_{2}$, and $I_{3}$ are the midpoints of $\overline{A_{3} G^{123}}, \overline{A_{2} G^{123}}$ and $\overline{A_{1} G^{123}}$ respectively.

Proof. Since $A_{1} \equiv\left[1: a_{1}^{2}: 2 a_{1}\right]$ and $G^{123} \equiv$ $\left[3: a_{1} a_{2}+a_{2} a_{3}+a_{1} a_{3}: 2\left(a_{1}+a_{2}+a_{3}\right)\right]$ we can check directly that the midpoint of $\overline{A_{1} G^{123}}$ is

$I_{1}=\left[6: a_{1} a_{2}+a_{2} a_{3}+a_{3} a_{1}+3 a_{1}^{2}: 2\left(4 a_{1}+a_{2}+a_{3}\right)\right]$.

Similarly, the midpoints of $\overline{A_{2} G^{123}}$ and $\overline{A_{3} G^{123}}$ are $I_{2}$ and $I_{3}$ respectively.

Theorem 5 The three lines $G_{23}^{2} G_{23}^{3}, G_{13}^{1} G_{13}^{3}$ and $G_{12}^{1} G_{12}^{2}$ are parallel to the tangents to the parabola at $A_{1}, A_{2}$ and $A_{3}$ respectively. These three lines are concurrent at the point $G_{123}$.

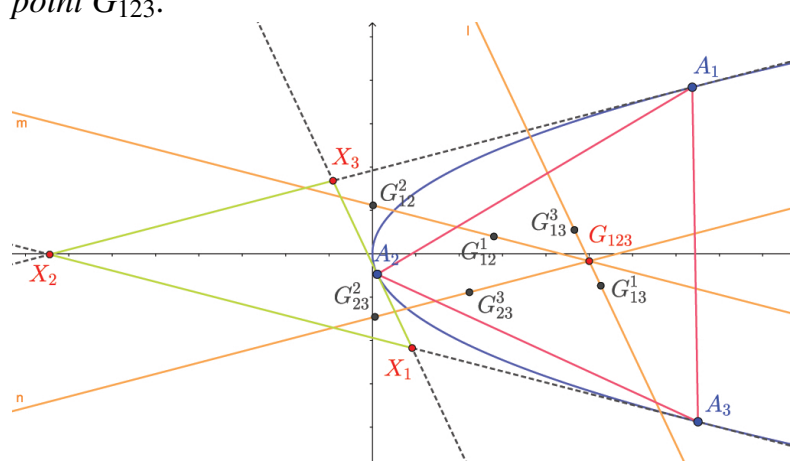

Figure 4: Parallel lines to tangents concurrent at $G_{123}$

Proof. From the formulas for the centroids we get

$G_{12}^{1} G_{12}^{2}=\left(a_{3}^{2}+2 a_{2} a_{3}+2 a_{1} a_{3}-a_{1}^{2}-a_{2}^{2}: 3:-3 a_{3}\right)$.

This is parallel to the tangent to the parabola at $A_{3}$ which is

$X_{1} X_{2}=\left(a_{3}^{2}: 1:-a_{3}\right)$.

To check that the centroid

$G_{123}=\left[3: a_{1}^{2}+a_{2}^{2}+a_{3}^{2}: 2\left(a_{1}+a_{2}+a_{3}\right)\right]$ is incident with this line we compute

$$
\begin{aligned}
& \left(a_{3}^{2}+2 a_{2} a_{3}+2 a_{1} a_{3}-a_{1}^{2}-a_{2}^{2}\right) 3+3\left(a_{1}^{2}+a_{2}^{2}+a_{3}^{2}\right) \\
& -3 a_{3} 2\left(a_{1}+a_{2}+a_{3}\right)=0 .
\end{aligned}
$$

By symmetry $G_{123}$ also lies on the lines $G_{23}^{2} G_{23}^{3}$ and $G_{13}^{1} G_{13}^{3}$, hence all three lines are concurrent.

Theorem 6 The three lines $G_{1}^{12} G_{1}^{13}, G_{2}^{23} G_{2}^{12}$ and $G_{3}^{13} G_{3}^{23}$ are parallel to the tangents to the parabola at $A_{1}, A_{2}$ and $A_{3}$ respectively. These three lines are concurrent at the point $G^{123}$.

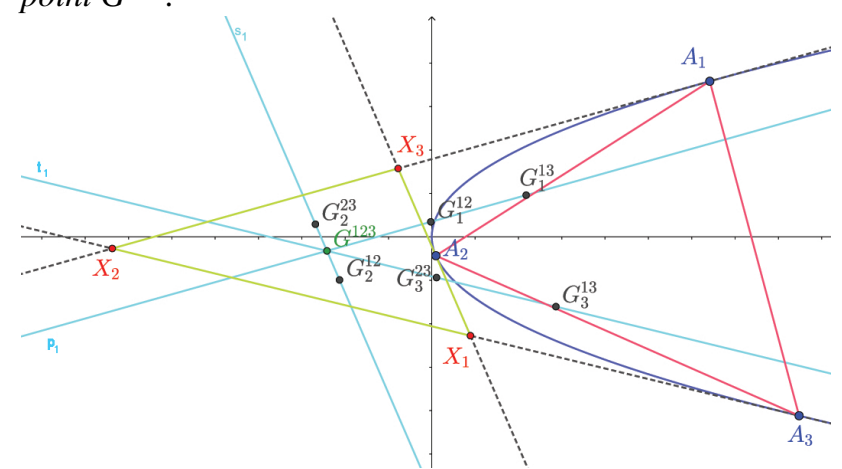

Figure 5: Another concurrency of parallel lines at $G^{123}$

Proof. From the formulas for the centroids we get

$G_{1}^{12} G_{1}^{13}=\left(2 a_{1}^{2}+a_{1} a_{2}+a_{1} a_{3}-a_{2} a_{3}: 3:-3 a_{1}\right)$.

This is again parallel to $X_{2} X_{3}=\left(a_{1}^{2}: 1:-a_{1}\right)$. And the centroid $G^{123}=\left[3: a_{1} a_{2}+a_{2} a_{3}+a_{3} a_{1}: 2\left(a_{1}+a_{2}+a_{3}\right)\right]$ is incident with $G_{1}^{12} G_{1}^{13}$ since

$$
\begin{aligned}
& \left(2 a_{1}^{2}+a_{1} a_{2}+a_{1} a_{3}-a_{2} a_{3}\right) 3+3\left(a_{1} a_{2}+a_{2} a_{3}+a_{3} a_{1}\right) \\
& -3 a_{1} 2\left(a_{1}+a_{2}+a_{3}\right)=0 .
\end{aligned}
$$

By symmetry $G^{123}$ also lies on the lines $G_{2}^{23} G_{2}^{12}$ and $G_{3}^{13} G_{3}^{23}$, hence all three lines are concurrent.

Theorem 7 Let $J_{1}$ be the meet of the lines $G_{13}^{3} G_{23}^{3}$ and $G_{12}^{2} G_{23}^{2}, J_{2}$ be the meet of the lines $G_{12}^{1} G_{13}^{1}$ and $G_{13}^{3} G_{23}^{3}$, and $J_{3}$ be the meet of the lines $G_{12}^{1} G_{13}^{1}$ and $G_{12}^{2} G_{23}^{2}$. Then for $1 \leq i<j \leq 3$

$\overrightarrow{J_{i} J_{j}}=-\frac{1}{2} \overrightarrow{A_{i} A_{j}}$.

Proof. The equations of the relevant lines are

$G_{12}^{1} G_{13}^{1}=$

$\left(\left(a_{2}^{2}+a_{3}^{2}-2 a_{1}^{2}+2 a_{1} a_{2}+2 a_{2} a_{3}+2 a_{3} a_{1}\right): 6:-3\left(a_{2}+a_{3}\right)\right)$

$G_{12}^{2} G_{23}^{2}=$

$\left(\left(a_{1}^{2}+a_{3}^{2}-2 a_{2}^{2}+2 a_{1} a_{2}+2 a_{2} a_{3}+2 a_{3} a_{1}\right): 6:-3\left(a_{1}+a_{3}\right)\right)$

$G_{13}^{3} G_{23}^{3}=$

$\left(\left(a_{1}^{2}+a_{2}^{2}-2 a_{3}^{2}+2 a_{1} a_{2}+2 a_{2} a_{3}+2 a_{3} a_{1}\right): 6:-3\left(a_{1}+a_{2}\right)\right)$. 
The meets of the lines can then be computed to be

$$
\begin{aligned}
J_{1} & =\left(G_{13}^{3} G_{23}^{3}\right)\left(G_{12}^{2} G_{23}^{2}\right) \\
& =\left[6:\left(2 a_{2}^{2}+2 a_{3}^{2}-a_{1}^{2}+a_{1} a_{2}+a_{2} a_{3}+a_{3} a_{1}\right): 6\left(a_{2}+a_{3}\right)\right] \\
J_{2} & =\left(G_{12}^{1} G_{13}^{1}\right)\left(G_{13}^{3} G_{23}^{3}\right) \\
& =\left[6:\left(2 a_{1}^{2}+2 a_{3}^{2}-a_{2}^{2}+a_{1} a_{2}+a_{2} a_{3}+a_{3} a_{1}\right): 6\left(a_{1}+a_{3}\right)\right] \\
J_{3} & =\left(G_{12}^{1} G_{13}^{1}\right)\left(G_{12}^{2} G_{23}^{2}\right) \\
& =\left[6:\left(2 a_{1}^{2}+2 a_{2}^{2}-a_{3}^{2}+a_{1} a_{2}+a_{2} a_{3}+a_{3} a_{1}\right): 6\left(a_{1}+a_{2}\right)\right] .
\end{aligned}
$$

Then using affine coordinates

$$
\begin{aligned}
\overrightarrow{J_{1} J_{2}} & =\left[\frac{1}{6}\left(2 a_{1}^{2}+2 a_{3}^{2}-a_{2}^{2}+a_{1} a_{2}+a_{2} a_{3}+a_{3} a_{1}\right),\left(a_{1}+a_{3}\right)\right] \\
& -\left[\frac{1}{6}\left(2 a_{2}^{2}+2 a_{3}^{2}-a_{1}^{2}+a_{1} a_{2}+a_{2} a_{3}+a_{3} a_{1}\right),\left(a_{2}+a_{3}\right)\right] \\
& =-\frac{1}{2}\left(a_{2}-a_{1}\right)\left(a_{1}+a_{2}, 2\right) \\
& =-\frac{1}{2} \overrightarrow{A_{1} A_{2}} .
\end{aligned}
$$

The other cases are symmetrically similar.

Corollary 3 The triangles $\Delta_{123}$ and $\overline{J_{1} J_{2} J_{3}}$ have parallel sides and so are similar, and so the signed areas satisfy

$s\left(\overrightarrow{A_{1} A_{2} A_{3}}\right): s\left(\overrightarrow{J_{1} J_{2} J_{3}}\right)=-4: 1$.

Proof. The argument is the same as previously, except now the orientations are reversed.

\section{The point $P$}

Theorem $8 \overline{J_{1} J_{2} J_{3}}$ is in perspective with $\Delta_{123}$. We denote the point of perspectivity by $P$. Then $P$ divides each of the sides $\overline{A_{1} J_{1}}, \overline{A_{2} J_{2}}$ and $\overline{A_{3} J_{3}}$ in the ratio $2: 1$, that is

$P=\frac{1}{3} A_{1}+\frac{2}{3} J_{1}=\frac{1}{3} A_{2}+\frac{2}{3} J_{2}=\frac{1}{3} A_{3}+\frac{2}{3} J_{3}$.

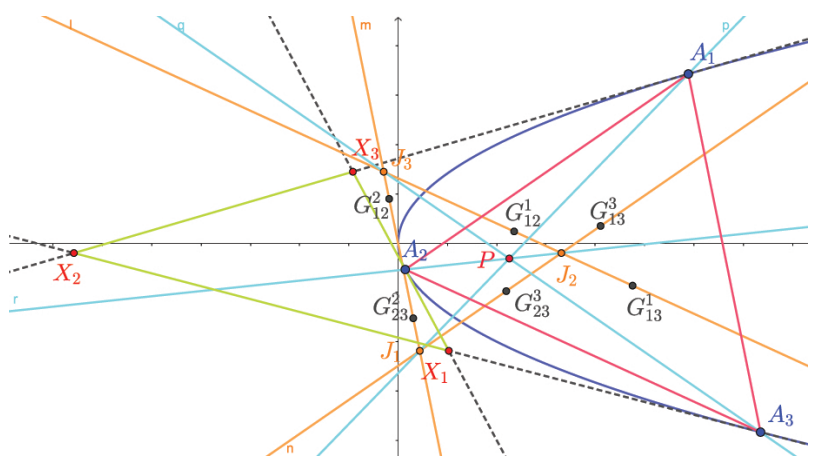

Figure 6: Perspectivity between $\overline{J_{1} J_{2} J_{3}}$ and $\overline{A_{1} A_{2} A_{3}}$ via $P$

Proof. We compute that the meet of $A_{1} J_{1}$ and $A_{2} J_{2}$ is the point

$P=\left[9: 2 a_{1}^{2}+2 a_{2}^{2}+2 a_{3}^{2}+a_{1} a_{2}+a_{2} a_{3}+a_{3} a_{1}: 6\left(a_{1}+a_{2}+a_{3}\right)\right]$
Moving to affine coordinates, we compute the affine combination

$$
\begin{aligned}
& \frac{1}{3} A_{1}+\frac{2}{3} J_{1}=\frac{1}{3}\left[a_{1}^{2}, 2 a_{1}\right] \\
& +\frac{2}{3} \cdot \frac{1}{6}\left[\left(2 a_{2}^{2}+2 a_{3}^{2}-a_{1}^{2}+a_{1} a_{2}+a_{2} a_{3}+a_{3} a_{1}\right), 6\left(a_{2}+a_{3}\right)\right] \\
& =\frac{1}{9}\left[2 a_{1}^{2}+2 a_{2}^{2}+2 a_{3}^{2}+a_{1} a_{2}+a_{1} a_{3}+a_{2} a_{3}, 6\left(a_{1}+a_{2}+a_{3}\right)\right] \\
& =P .
\end{aligned}
$$

The other ratios

$P=\frac{1}{3} A_{2}+\frac{2}{3} J_{2}=\frac{1}{3} A_{3}+\frac{2}{3} J_{3}$

are similar.

Corollary 4 The triangles $\overline{I_{1} I_{2} I_{3}}$ and $\overline{J_{1} J_{2} J_{3}}$ are affinely negatively congruent, by which we mean that corresponding vectors of sides are negatives of each other.

Proof. This follows directly from the relations between the vectors of the sides of the triangles.

Theorem 9 The points $G_{123}, G^{123}$, and $P$ are collinear. $P$ also divides the side $\overline{G_{123} G^{123}}$ in the ratio $2: 1$, that is

$P=\frac{1}{3} G^{123}+\frac{2}{3} G_{123}$

Proof. Using the affine coordinates of the centroids we find that

$$
\begin{aligned}
& \frac{1}{3} G^{123}+\frac{2}{3} G_{123} \\
& =\frac{1}{3}\left[\frac{\left(a_{1} a_{2}+a_{2} a_{3}+a_{3} a_{1}\right)}{3}, \frac{2\left(a_{1}+a_{2}+a_{3}\right)}{3}\right] \\
& \quad+\frac{2}{3}\left[\frac{\left(a_{1}^{2}+a_{2}^{2}+a_{3}^{2}\right)}{3}, \frac{2\left(a_{1}+a_{2}+a_{3}\right)}{3}\right] \\
& =\frac{1}{9}\left[2 a_{1}^{2}+2 a_{2}^{2}+2 a_{3}^{2}+a_{1} a_{2}+a_{1} a_{3}+a_{2} a_{3}, 6\left(a_{1}+a_{2}+a_{3}\right)\right] \\
& =P .
\end{aligned}
$$

Theorem 10 The triangles $\overline{I_{1} I_{2} I_{3}}$ and $\overline{J_{1} J_{2} J_{3}}$ are perspective from the point $M$ which is the midpoint of $G_{123}$ and $G^{123}$.

Proof. The midpoint of $\overline{G_{123} G^{123}}$ is

$M=$

$\left[6: a_{1}^{2}+a_{2}^{2}+a_{3}^{2}+a_{1} a_{2}+a_{2} a_{3}+a_{3} a_{1}: 4\left(a_{1}+a_{2}+a_{3}\right)\right]$. 
Computing the lines $I_{1} J_{1}, I_{2} J_{2}$ and $I_{3} J_{3}$ we get

$$
\begin{gathered}
I_{1} J_{1}=\left(-3 a_{1} a_{2}^{2}+3 a_{1}^{2} a_{2}-3 a_{1} a_{3}^{2}+3 a_{1}^{2} a_{3}+2 a_{1}^{3}-a_{2}^{3}-a_{3}^{3}\right. \\
\left.\quad: 6\left(2 a_{1}-a_{2}-a_{3}\right): 3\left(-2 a_{1}^{2}+a_{2}^{2}+a_{3}^{2}\right)\right) \\
I_{2} J_{2}=\left(3 a_{1} a_{2}^{2}-3 a_{1}^{2} a_{2}-3 a_{2} a_{3}^{2}+3 a_{2}^{2} a_{3}-a_{1}^{3}+2 a_{2}^{3}-a_{3}^{3}\right. \\
\left.: 6\left(-a_{1}+2 a_{2}-a_{3}\right): 3\left(a_{1}^{2}-2 a_{2}^{2}+a_{3}^{2}\right)\right) \\
I_{3} J_{3}=\left(3 a_{1} a_{3}^{2}-3 a_{1}^{2} a_{3}+3 a_{2} a_{3}^{2}-3 a_{2}^{2} a_{3}-a_{1}^{3}-a_{2}^{3}+2 a_{3}^{3}\right. \\
\left.: 6\left(-a_{1}-a_{2}+2 a_{3}\right): 3\left(a_{1}^{2}+a_{2}^{2}-2 a_{3}^{2}\right)\right) .
\end{gathered}
$$

By computation the meet of $I_{1} J_{1}$ and $I_{2} J_{2}$ is:

$$
\begin{aligned}
& 9\left(a_{2}-a_{3}\right)\left(a_{1}-a_{3}\right)\left(a_{1}-a_{2}\right) \\
& {\left[6: a_{1}^{2}+a_{2}^{2}+a_{3}^{2}+a_{1} a_{2}+a_{1} a_{3}+a_{2} a_{3}: 4\left(a_{1}+a_{2}+a_{3}\right)\right]}
\end{aligned}
$$

which after re-scaling becomes

$$
\begin{aligned}
& M= \\
& {\left[6: a_{1}^{2}+a_{2}^{2}+a_{3}^{2}+a_{1} a_{2}+a_{1} a_{3}+a_{2} a_{3}: 4\left(a_{1}+a_{2}+a_{3}\right)\right] .}
\end{aligned}
$$

Theorem 11 The four points $G_{23}^{1}, G_{13}^{2}, G_{12}^{3}$ and $P$ are collinear.

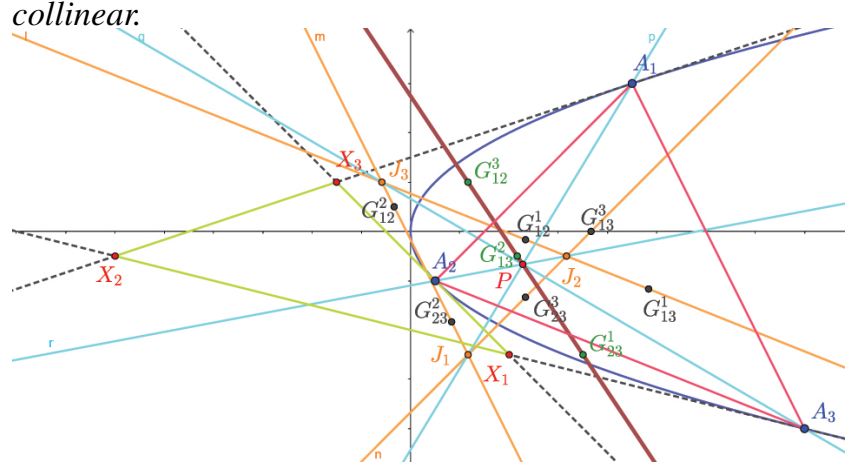

Figure 7: Collinearity of $G_{23}^{1}, G_{13}^{2}, G_{12}^{3}$ and $P$

Proof. The four points are

$$
\begin{aligned}
G_{23}^{1}= & {\left[3: a_{2}^{2}+a_{3}^{2}+a_{2} a_{3}: 3\left(a_{2}+a_{3}\right)\right] } \\
G_{13}^{2}= & {\left[3: a_{1}^{2}+a_{3}^{2}+a_{1} a_{3}: 3\left(a_{1}+a_{3}\right)\right] } \\
G_{12}^{3}= & {\left[3: a_{1}^{2}+a_{2}^{2}+a_{1} a_{2}: 3\left(a_{1}+a_{2}\right)\right] } \\
P= & {\left[9: 2 a_{1}^{2}+2 a_{2}^{2}+2 a_{3}^{2}+a_{1} a_{2}+a_{1} a_{3}+a_{2} a_{3}\right.} \\
& \left.: 6\left(a_{1}+a_{2}+a_{3}\right)\right] .
\end{aligned}
$$

The equation of the line

$G_{23}^{1} G_{13}^{2}$ is $\left[3:-\left(a_{1}+a_{2}+a_{3}\right): a_{1} a_{2}+a_{1} a_{3}+a_{2} a_{3}\right]$,

which passes through the points $G_{12}^{3}$ and $P$.

Theorem 12 The three points $G_{1}^{23}, G_{2}^{13}$ and $G_{3}^{12}$ are collinear on a line $l$, which is also parallel to the line through the points $G_{23}^{1}, G_{13}^{2}, G_{12}^{3}$ and P. Moreover $l$ is a tangent to the parabola at the point $Q$ where it meets the central line $G_{123} G^{123}$. Furthermore $Q$ divides the side $\overline{G^{123} G_{123}}$ in the ratio $1: 2$. So the four points $G^{123}, Q, P$ and $G_{123}$ are equally spaced on the central line.

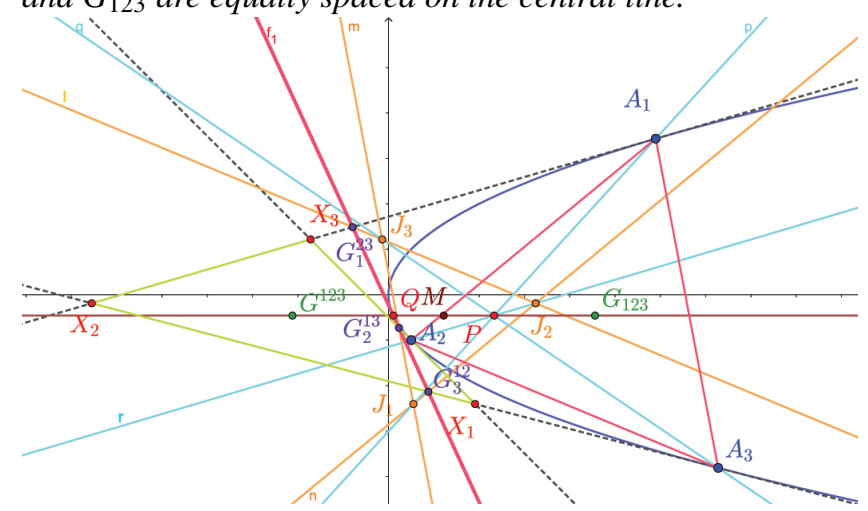

Figure 8: Equal spacing of $G^{123}, Q, P$ and $G_{123}$ and tangency at $Q$

Proof. The three points are

$G_{1}^{23}=\left[3: a_{1}\left(a_{1}+a_{2}+a_{3}\right): 4 a_{1}+a_{2}+a_{3}\right]$

$G_{2}^{13}=\left[3: a_{2}\left(a_{1}+a_{2}+a_{3}\right): a_{1}+4 a_{2}+a_{3}\right]$

$G_{3}^{12}=\left[3: a_{3}\left(a_{1}+a_{2}+a_{3}\right): a_{1}+a_{2}+4 a_{3}\right]$.

The equation of the line $G_{1}^{23} G_{2}^{13}$ is

$\left[\left(a_{1}+a_{2}+a_{3}\right)^{2}: 9:-3\left(a_{1}+a_{2}+a_{3}\right)\right]$,

which passes through the point $G_{3}^{12}$ by computation

$3\left(a_{1}+a_{2}+a_{3}\right)^{2}+9 a_{3}\left(a_{1}+a_{2}+a_{3}\right)$

$-3\left(a_{1}+a_{2}+a_{3}\right)\left(a_{1}+a_{2}+4 a_{3}\right)=0$.

Hence the three points $G_{1}^{23}, G_{2}^{13}$ and $G_{3}^{12}$ are collinear. This line is also a tangent to the parabola since it is in the form of $\left(a^{2}: 1:-a\right)$. Moreover it is parallel to the line through the points $G_{23}^{1}, G_{13}^{2}, G_{12}^{3}$ and $P$ :

$\left(a_{1} a_{2}+a_{1} a_{3}+a_{2} a_{3}: 3:-\left(a_{1}+a_{2}+a_{3}\right)\right)$.

The meet of the line $G_{1}^{23} G_{2}^{13}$ with the central line $G_{123} G^{123}$ is the point

$Q=\left[9:\left(a_{1}+a_{2}+a_{3}\right)^{2}: 6\left(a_{1}+a_{2}+a_{3}\right)\right]$

which lies on the parabola, and we can check that

$\frac{2}{3} G^{123}+\frac{1}{3} G_{123}$

$=\frac{2}{3}\left[\frac{\left(a_{1} a_{2}+a_{2} a_{3}+a_{3} a_{1}\right)}{3}, \frac{2\left(a_{1}+a_{2}+a_{3}\right)}{3}\right]$

$+\frac{1}{3}\left[\frac{\left(a_{1}^{2}+a_{2}^{2}+a_{3}^{2}\right)}{3}, \frac{2\left(a_{1}+a_{2}+a_{3}\right)}{3}\right]$

$=\frac{1}{9}\left[\left(a_{1}+a_{2}+a_{3}\right)^{2}, 6\left(a_{1}+a_{2}+a_{3}\right)\right]=Q$. 
Theorem $13 M$ is the midpoint of the following pair of points: $P$ and $Q, G_{123}$ and $G^{123}, I_{1}$ and $J_{1}, I_{2}$ and $J_{2}, I_{3}$ and $J_{3}, G_{23}^{1}$ and $G_{1}^{23}, G_{13}^{2}$ and $G_{2}^{13}, G_{12}^{3}$ and $G_{3}^{12}, G_{12}^{1}$ and $G_{3}^{23}, G_{23}^{3}$ and $G_{1}^{12}, G_{12}^{2}$ and $G_{3}^{13}, G_{13}^{3}$ and $G_{2}^{12}, G_{23}^{2}$ and $G_{1}^{13}$, $G_{13}^{1}$ and $G_{2}^{23}$.

Proof. From the explicit forms of the various points that we have so far determined, the midpoint of $\overline{I_{1} J_{1}}$ is

$$
\begin{aligned}
& \frac{1}{2}\left[\frac{a_{1} a_{2}+a_{2} a_{3}+a_{3} a_{1}+3 a_{1}^{2}}{6}, \frac{2\left(a_{2}+a_{3}+4 a_{1}\right)}{6}\right] \\
& +\frac{1}{2}\left[\frac{\left(2 a_{2}^{2}+2 a_{3}^{2}-a_{1}^{2}+a_{1} a_{2}+a_{1} a_{3}+a_{2} a_{3}\right)}{6}, \frac{6\left(a_{2}+a_{3}\right)}{6}\right] \\
& =\left[\frac{a_{1}^{2}+a_{2}^{2}+a_{3}^{2}+a_{1} a_{2}+a_{1} a_{3}+a_{2} a_{3}}{6}, \frac{4\left(a_{1}+a_{2}+a_{3}\right)}{6}\right] \\
& =M
\end{aligned}
$$

and similarly we can verify that the midpoint of all the other pairs are also $M$.

Corollary 5 The lines $P Q, G_{123} G^{123}, I_{1} J_{1}, I_{2} J_{2}, I_{3} J_{3}$, $G_{23}^{1} G_{1}^{23}, \quad G_{13}^{2} G_{2}^{13}, \quad G_{12}^{3} G_{3}^{12}, \quad G_{12}^{1} G_{3}^{23}, \quad G_{23}^{3} G_{1}^{12}, G_{12}^{2} G_{3}^{13}$, $G_{13}^{3} G_{2}^{12}, G_{23}^{2} G_{1}^{13}$ and $G_{13}^{1} G_{2}^{23}$ are all concurrent at the point $M$.

Proof. Since $M$ is the midpoint of all the intervals, clearly the lines are all concurrent at $M$.

Corollary 6 The quadrilateral formed by any pairs of the diagonals from the set

$$
\begin{gathered}
\left\{P Q, G_{123} G^{123}, I_{1} J_{1}, I_{2} J_{2}, I_{3} J_{3}, G_{23}^{1} G_{1}^{23}, G_{13}^{2} G_{2}^{13}, G_{12}^{3} G_{3}^{12},\right. \\
\left.G_{12}^{1} G_{3}^{23}, G_{23}^{3} G_{1}^{12}, G_{12}^{2} G_{3}^{13}, G_{13}^{3} G_{2}^{12}, G_{23}^{2} G_{1}^{13}, G_{13}^{1} G_{2}^{23}\right\}
\end{gathered}
$$

is a parallelogram.

Proof. Since all the diagonals from the set

$$
\begin{gathered}
\left\{P Q, G_{123} G^{123}, I_{1} J_{1}, I_{2} J_{2}, I_{3} J_{3}, G_{23}^{1} G_{1}^{23}, G_{13}^{2} G_{2}^{13}, G_{12}^{3} G_{3}^{12},\right. \\
\left.G_{12}^{1} G_{3}^{23}, G_{23}^{3} G_{1}^{12}, G_{12}^{2} G_{3}^{13}, G_{13}^{3} G_{2}^{12}, G_{23}^{2} G_{1}^{13}, G_{13}^{1} G_{2}^{23}\right\}
\end{gathered}
$$

share a common midpoint $M$, the quadrilateral formed by any pairs of the diagonals will result in a parallelogram. Counting we see that from this list there will be $14 \times 13 / 2=91$ different parallelograms.

\section{Further directions}

The results of this paper are affine results, because they rest on notions of midpoints and centroids, but no metrical structure. However it is not hard to generalize this to projective geometry if we remember that any line in the projective plane can act as the distinguished 'line at infinity' which characterizes affine geometry, and that then the usual midpoint construction is generalized to harmonic conjugation of the distinguished point at infinity on the line determined by two points. In this way we can restate the results above in projective geometry, but for a conic which is tangent to this line at infinity. Somewhat remarkably, our investigations suggest a rather big generalization of this, which we state as a conjecture.

Conjecture: All of the results of this paper that do not involve the axis of the parabola hold if the parabola is replaced by a generic conic.

However the relative ease of proof that we have been able to obtain with the standard coordinates on the parabola will have to be rethought.

\section{References}

[1] S .C. Choi, N. J. Wildberger, The Universal Parabola, KoG 22 (2018), 24-40.

[2] T. E. FAulkner, Projective Geometry (2nd ed.), Oliver and Boyd, London, 1952.

[3] D. LIU, Centroids of Triangles on a Parabola, at https://brilliant.org/discussions/thread/ centroids-of-triangles-on-a-parabola/

[4] G. Salmon, A Treatise on Conic Sections (6th ed.), Longmans, Green, and Co., London, 1879.

[5] D. M. Y. Sommerville, Analytical Conics, G. Bell, London, 1933.

[6] B. Spain, Analytical conics, Pergamon Press, London, 1957.

[7] J. A. TodD, Projective and Analytical Geometry, Sir Isaac Pitman \& Sons, LTD, London, 1960.

[8] I. Todhunter, A Treatise on Plane Co-ordinate Geometry as applied to the straight line and the conic sections (4th ed.), Macmillan, London, 1867. 


\section{Si Chun Choi}

orcid.org/0000-0003-3856-0751

e-mail: si.choi@det.nsw.edu.au

Caringbah High School

Sydney, Australia

\section{N. J. Wildberger}

orcid.org/0000-0003-3503-6495

e-mail: n.wildberger@unsw.edu.au

School of Mathematics and Statistics UNSW

Sydney, Australia 